C. J. Greenbaum - G. Eisenbarth • M. Atkinson •

L. Yu · S. Babu • D. Schatz - A. Zeidler · T. Orban •

C. Wasserfall $\cdot$ D. Cuthbertson $\cdot$ J. Krischer $\cdot$ DPT-1

study group

\title{
High frequency of abnormal glucose tolerance in DQA1*0102/ DQB1* 0602 relatives identified as part of the Diabetes Prevention Trial-Type 1 Diabetes
}

Received: 27 February 2004 / Accepted: 9 August 2004 / Published online: 16 December 2004

(C) Springer-Verlag 2004

\begin{abstract}
Aims/hypothesis: Immunological and genetic markers can be used to assess risk of developing type 1 diabetes prior to the onset of clinical symptoms. Autoantibody-positive relatives of patients with type 1 diabetes are at increased risk for disease, while the presence of HLA $D Q A 1^{*} 0102 / D Q B 1^{*} 0602$ is thought to confer protection. Using the unique population identified by the Diabetes Prevention Trial-Type Diabetes (DPT-1), our aim was to determine if these individuals were protected from type 1 diabetes. Methods: We described metabolic and immunological characteristics of islet cell cytoplasmic autoantibodies-positive relatives with $D Q B 1 * 0602$ identified as part of DPT- 1 . Results: We found that $32 \%$ of $D Q B 1 * 0602$-positive relatives identified through the DPT1 had abnormalities of glucose tolerance despite the fact that only $19 \%$ had multiple type 1 diabetes-associated autoantibodies and only $13 \%$ had abnormal insulin secretion,
\end{abstract}

C. J. Greenbaum $(\bowtie)$

Benaroya Research Institute at Virginia Mason,

1201 9th Ave,

Seattle, WA, 98101, USA

e-mail: cjgreen@benaroyaresearch.org

Tel.: +1-206-5155232

Fax: +1-206-5155239

G. Eisenbarth $\cdot$ L. Yu $\cdot$ S. Babu

Barbara Davis Center for Childhood Diabetes,

Denver, CO, USA

M. Atkinson · D. Schatz · C. Wasserfall

University of Florida,

Gainesville, FL, USA

A. Zeidler

University of Southern California,

Los Angeles, CA, USA

T. Orban

Joslin Diabetes Center,

Boston, MA, USA

D. Cuthbertson · J. Krischer

University of South Florida,

Tampa, FL, USA markers typically associated with the disease. In addition, these markers were not associated with abnormal glucose tolerance. In contrast, the $D Q B 1 * 0602$-positive relatives had elevated fasting insulin $(117 \pm 10 \mathrm{pmol} / \mathrm{l})$ and homeostasis model assessment of insulin resistance (HOMA-R) $(4.90 \pm 0.5)$ values, which are more commonly associated with type 2 diabetes. The later marker of insulin resistance was associated with glucose tolerance status. Conclusions/ interpretation: Our data indicate that $D Q A 1^{*} 0102 /$ $D Q B 1^{*} 0602$ relatives identified through DPT-1 have a high frequency of abnormal glucose tolerance and a disease phenotype with characteristics of type 1 and type 2 diabetes. Thus, multiple pathways to abnormal glucose tolerance are present within families of these type 1 patients.

Keywords Aetiology · Human leucocyte antigen · Insulin resistance $\cdot$ Pathophysiology $\cdot$ Prevention

Abbreviations DASP: Diabetes Antibody

Standardization Program - DPT-1: Diabetes Prevention Trial-Type 1 Diabetes · FPIR: First-phase insulin release - GADA: Glutamic acid decarboxylase antibodies . HOMA-B: Homeostasis model assessment of beta cell function - HOMA-R: Homeostasis model assessment of insulin resistance $\cdot$ ICA: islet cell cytoplasmic autoantibodies - LADA: latent autoimmune diabetes of adults

\section{Introduction}

Type 1 diabetes is an autoimmune disease whose natural history and pathogenesis can be assessed prior to symptomatic onset through a combination of genetic and humoral immune markers. Indeed, genes from the $H L A$ $D R$ and $D Q$ region confer both disease susceptibility and resistance, while autoantibodies against various islet cell autoantigens normally mark ongoing anti-beta-cell immunity and increased risk of developing the disease. We 
previously described a population of autoantibody-positive relatives of persons with type 1 diabetes who have the HLA haplotype $D Q A 1 * 0102 / D Q B 1 * 0602$ [1]. Though the presence of autoantibodies usually suggests an increased risk for type 1 diabetes, the presence of $D Q A 1^{*} 0102 /$ $D Q B 1 * 0602$ is thought to impart protection from type 1 diabetes, since it is only present in approximately $1 \%$ to $3 \%$ of Caucasian patients with the disease, compared with a $25 \%$ frequency in the general population [2-7]. In addition, previous reports analysing a small number of similarly autoantibody-positive relatives led to the concept that the presence of $D Q A 1^{*} 0102 / D Q B 1^{*} 0602$ affords dominant protection from type 1 diabetes [8-10].

Based on the ability to use genetic and autoantibody markers to assess future risk for type 1 diabetes, the Diabetes Prevention Trial-Type Diabetes (DPT-1) was established to observe whether antigen-specific therapies could delay the onset of disease in nondiabetic relatives of individuals with type 1 diabetes who were deemed at increased risk of the disease [11]. As part of this screening process, the DPT- 1 identified a large number of $D Q B 1^{*} 0602$-positive subjects. Using data from an initial 100 subjects, we previously confirmed by sequence analysis that these subjects were in fact $D Q B 1^{*} 0602$ [12] and reported that these relatives were much less likely to be confirmed positive for islet cell cytoplasmic autoantibodies (ICA), to have multiple antibodies, and to have low firstphase insulin release [1]. In this report, we extend our previous cross-sectional observations and present additional autoantibody and metabolic data that address the natural history of these two parameters in this population of subjects. Specifically, the aim of our study was to determine whether $D Q B 1^{*} 0602$-positive relatives identified through the DPT- 1 are protected from type 1 diabetes.

\section{Materials and methods}

These investigations were approved by the institutional review boards at participating centres prior to study initiation and informed consent was obtained from all individuals and/or their parent/guardian prior to participation.

\section{Subjects}

An overview of subject selection and treatments is given in Fig. 1.

Antibody-positive DQA1*0102/DQB1*0602 subjects From February 1994 through to May 2000, the DPT-1 screened more than 81,000 first- and second-degree relatives of patients with type 1 diabetes, of whom $3.6 \%$ were identified as positive for ICA [11]. All individuals found ICA-positive at screening were offered the opportunity to participate in further studies including that of HLA assessment. Autoantibody-positive relatives identified with $D Q A 1 * 0102 / D Q B 1 * 0602$ were excluded from further participation in the DPT-1 because their risk of
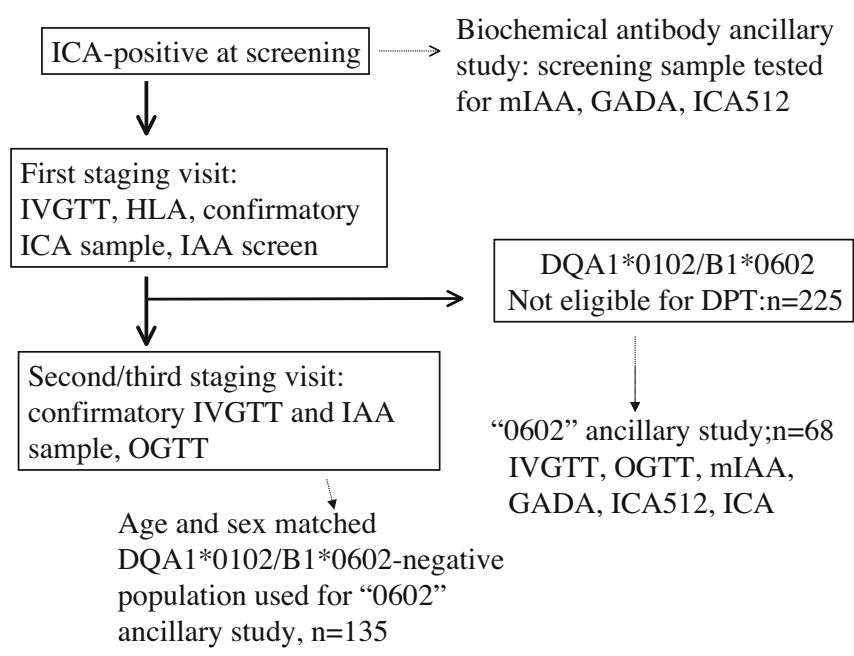

Fig. 1 Source of subjects and data. Boxed text and solid arrows delineate DPT-1 protocol visits and tests. Unboxed text and dotted arrows delineate the ancillary study populations and data

developing type 1 diabetes was deemed too low for a trial whose principal goal was to assess therapeutic interventions. From 1994 to 2001 DPT-1 screening identified 225 ICA-positive relatives with the $D Q A 1 * 0102 / D Q B 1 * 0602$ haplotype. As part of the DPT-1, these subjects were notified that they had a low risk for type 1 diabetes.

The ancillary study, which formed the basis of this current report, began in June 1999. Our original study design planned to recruit 60 subjects. Of the 135 subjects contacted, 68 agreed to travel to Seattle, Washington, to undergo OGTT, IVGTT, and sampling for additional autoantibody markers often associated with type 1 diabetes. The mean age of these subjects at the time of OGTT testing was $21.0 \pm 1.4$ years (range $5.6-48.5$ years). The male to female ratio was $1: 1$. There were 52 first-degree relatives of individuals with type 1 diabetes $(22$ siblings, 23 offspring, 7 parents), the remainder were second-degree relatives. Of the subjects studied, 58 were non-Hispanic whites, 3 were blacks, 2 Hispanics, 1 American Indian and 1 Pacific Islander. In three subjects ethnicity was unspecified. The ethnicity, sex, age, and relationship to proband of this group did not differ from the remaining $157 D Q A 1 * 0102 / D Q B 1 * 0602$ subjects identified, who did not participate in this ancillary study.

Each of these 68 subjects had a first- or second-degree proband with type 1 diabetes which made them eligible for DPT-1 screening. HLA typing was performed on 66 of these type 1 diabetic probands.

Autoantibody-positive DQA1*0102/DQB1*0602-negative subjects Data from relatives who did not have HLA $D Q A 1 * 0102 / D Q B 1 * 0602$ and which had been obtained as part of the DPT-1 were used for comparative purposes. Subjects who underwent OGTT as part of DPT-1 were matched for age within 5 years and sex in a 2:1 ratio with the prospectively followed subjects $(n=135)$. There were 124 non-Hispanic whites, 2 blacks, 5 Hispanics, 1 Asian/ Pacific Islander, and 4 of other or unspecified ethnicity in 
this matched cohort. This distribution was no different than that of the $D Q A 1 * 0102 / D Q B 1 * 0602$ subjects $(p=0.5)$.

\section{Autoantibodies}

Islet cell cytoplasmic autoantibodies Islet cell cytoplasmic autoantibodies for DPT-1 were determined by immunofluorescence assay on frozen sections of human pancreas in the DPT-1 ICA Core Laboratories (Gainesville, FL, USA; February 1994 to September 1997; New Orleans, LA, USA; September 1997 to January, 1999; Gainesville, FL, USA; January 1999 to 2001). ICA values of 10 or more Juvenile Diabetes Foundation (JDF) units were considered positive. In the 1995 Immunology of Diabetes Society workshop, this ICA assay had a specificity of $100 \%$ and a sensitivity of $74.4 \%$ for new-onset type 1 diabetes patients who were younger than 30 years of age [13].

GADA, ICA512A and mIAA Autoantibodies against glutamic acid decarboxylase (GADA), ICA512 (ICA512A), insulin (micro IAA [mIAA]) were determined in Denver, CO, USA, on DPT-1 samples as part of a DPT-1 ancillary study. As previously described, a combined GADA and ICA512A radioassay was performed [14]. Labelled recombinant GAD65 and ICA512 were produced by in vitro transcription/translation with differential labelling $\left(\left[{ }^{3} \mathrm{H}\right]\right.$ GAD65 and $\left.\left[{ }^{35} \mathrm{~S}\right] \mathrm{ICA} 512\right)$. The levels of both autoantibodies were expressed as an index value. The interassay coefficients of variation were $6 \%$ and $9.6 \%$ for GADA and ICA512A respectively. The upper limits of normal (0.032 for GADA; 0.049 for ICA512A) were established as the 99th percentile for GADA and for ICA512A from receiver operating characteristics curves in 198 healthy control subjects and 50 patients with new-onset type 1 diabetes. In the 2002 Diabetes Antibody Standardization Program (DASP) proficiency testing, the sensitivity for the GADA assay was $90 \%$ with a specificity of $93 \%$. The sensitivity for the ICA512A assay was $62 \%$ with a specificity of $99 \%$. The mIAA assay is distinct from the IAA assay used as entry criteria in DPT- 1 . The interassay coefficient of variation for the mIAA assay was $12 \%$. In the aforementioned DASP testing, the sensitivity for the mIAA assay was $62 \%$ with $98 \%$ specificity.

\section{Measures of beta cell function}

Oral glucose tolerance Oral glucose tolerance tests were performed after an overnight fast and insertion of an i.v. catheter in an antecubital vein. Samples were drawn at -10 and $0 \mathrm{~min}$. Oral glucose was administered in a dose of $1.75 \mathrm{~g} / \mathrm{kg}$ body weight to a maximum of $75 \mathrm{~g}$ as a solution in flavoured water and consumed within $5 \mathrm{~min}$. Blood samples were obtained at 30,60, 90 and $120 \mathrm{~min}$ after glucose consumption to determine glucose, insulin and Cpeptide levels. The American Diabetes Association's definitions were used to define categories of glucose tolerance on OGTT [15]. Thus, normal glucose tolerance was defined as fasting glucose lower than $6.1 \mathrm{mmol} / \mathrm{l}$ and 2-h glucose $7.8 \mathrm{mmol} / \mathrm{l}$ or less. Abnormal glucose tolerance included subjects with diabetes (mean fasting glucose $\geq 7 \mathrm{mmol} / 1$ and/or 2 -h value $\geq 11.1 \mathrm{mmol} / \mathrm{l}$ ), IFG (fasting value between 6.1 and $7.0 \mathrm{mmol} / \mathrm{l}$ with a 2 -h value $<7.8 \mathrm{mmol} / \mathrm{l}$ ), or IGT (2-h value between 7.8 and 11.0 $\mathrm{mmol} / \mathrm{l})$.

First-phase insulin secretion Intravenous glucose tolerance tests were performed according to the ICARUS protocol $[16,17]$. After an overnight fast and insertion of an i.v. catheter in an antecubital vein, samples were drawn at -10 and -4 min. Fasting insulin was determined from a mean of these two baseline values. A solution of $25 \%$ glucose $(0.5 \mathrm{~g} / \mathrm{kg}$; maximum $35 \mathrm{~g})$ was then administered i.v. over a 3-min period. After the infusion of glucose, samples were drawn at 1, 3, 5, 7 and $10 \mathrm{~min}$. Insulin and glucose were measured in the DPT-1 Beta Cell Function Core Laboratory (Seattle, WA, USA). The insulin values at 1 and $3 \mathrm{~min}$ were added to calculate a measure of firstphase insulin release (FPIR). The tenth percentile of normal controls for siblings and offspring over age 8 years was $600 \mathrm{pmol} / \mathrm{l}$. The tenth percentile of normal controls for siblings and offspring under age 8 years and the first percentile for parents was $360 \mathrm{pmol} / \mathrm{l}$. These values were determined from intravenous glucose tolerance test studies of 224 nondiabetic individuals aged 4 to 45 years of age and were used as the thresholds for eligibility for the parenteral insulin intervention trial [11].

Homeostasis model assessment of beta cell function (HOMA-B) This index of resting beta cell function correlates with beta cell secretion measured by intravenous glucose tolerance and was calculated as follows: fasting insulin $(\mu \mathrm{U} / \mathrm{ml}) \times 20 /($ fasting glucose $[\mathrm{mmol}-3.5])[18,19]$.

Measures of insulin resistance

Homeostasis model assessment of insulin resistance $(H O M A-R)$ This index, derived from fasting glucose and insulin values, correlates with both the hyperglycaemic or euglycaemic, hyperinsulinaemic clamp and minimal model measures of insulin resistance. It was calculated as follows: (fasting insulin $[\mu \mathrm{U} / \mathrm{ml}] \times$ fasting glucose $[\mathrm{mmol} / 1] / 22.5$ ) [18-20].

HLA typing Samples for HLA typing were obtained during the first staging visit for ICA-positive relatives. HLA typing was performed at the DPT-1 HLA Core Laboratory (Denver, CO, USA). HLA-DQA1 and DQB1 alleles were typed using PCR and sequence-specific oligonucleotide probes $[21,22]$. HLA DQA1*0102, DQB1*0602-positive and -negative control samples were included in all assays. 
Table 1 Characteristics of $D Q A 1 * 0102 / D Q B 1 * 0602$-positive and -negative relatives

\begin{tabular}{lccc}
\hline & $D Q B 1 * 0602$-positive $(n=68)$ & $D Q B 1 * 0602$-negative $(n=135)$ & $p$ value \\
\hline Abnormal glucose tolerance $(\%)$ & 32.4 & 17.0 & 0.01 \\
Fasting insulin (pmol/l; mean \pm SE) & $117 \pm 10$ & $80 \pm 4$ & 0.0001 \\
HOMA-R (mean $\pm \mathrm{SE})$ & $4.90 \pm 0.5$ & $3.17 \pm 0.3$ & 0.001 \\
$\mathrm{BMI}\left(\mathrm{kg} / \mathrm{m}^{2}\right.$; mean $\left.\pm \mathrm{SE}\right)$ & $24.2 \pm 0.9$ & $23.3 \pm 0.5$ & 0.3 \\
$\mathrm{HbA}_{1} \mathrm{c}(\mathrm{mean} \pm \mathrm{SE})$ & $5.4 \pm 0.08$ & $5.3 \pm 0.04$ & 0.1 \\
\hline
\end{tabular}

Statistical analysis

Categorical variables were compared between those with and without $D Q A 1 * 0102 / D Q B 1 * 0602$ by the Pearson's chi-square test or Fisher's exact test (depending on the number of individuals per cell). Continuous variables were compared by the $t$-test or Wilcoxon rank-sum test (depending on the distribution of the variable). Values for HOMA-B and HOMA-R were log transformed for analysis. With the exception of data in Table 2, tests of significance were two tailed and a $p$ value of 0.05 was considered significant. In Table 2, since multiple comparisons were done, none of the differences between variables are considered significant. Statistical analyses were performed using SAS software (SAS Institute, Cary, NC, USA).

\section{Results}

Immunological and genetic assessments DQA1*0102/ $D Q B 1 * 0602$ ICA-positive subjects had GADA, ICA512A, and mIAA autoantibody testing performed on either their initial DPT-1 screening sample or on a subsequent sample obtained as part of the prospective study. Using data from the first available result, 12/68 $(17.7 \%)$ subjects had GADA, $3 / 68$ (4.4\%) subjects were ICA512A-positive, and 4/67 (6.0\%) were mIAA-positive.

HLA typing revealed that $2 / 66(3 \%)$ of the subjects have probands with type 1 diabetes who also have the $D Q B 1^{*} 0602$ haplotype. This was not different than the frequency of $D Q B 1^{*} 0602$ seen among a general population of people with diabetes 49/1664 (2.9\%) or among GADA or ICA512A-positive individuals with diabetes (14/970 [1.4\%]; G. Eisenbarth, unpublished observation).
Metabolic assessments OGTT results consistent with the diagnosis of diabetes were found in 2/68 (2.9\%) of subjects. Results in 19 subjects indicated IGT, and one subject had IFG. Thus, 22/68 (32.4\%) of DQB1*0602 relatives had abnormalities of glucose tolerance, significantly more than the $23 / 135(17.0 \%)$ of relatives without $D Q B 1 * 0602(p=0.012)$ (Table 1). Of these 23 glucoseintolerant relatives without $D Q A 1 * 0102 / D Q B 1 * 0602,4$ had OGTT results consistent with the diagnosis of diabetes, 3 had IFG, and 16 had IGT.

With regard to measures associated with insulin resistance, the $D Q B 1^{*} 0602$ subjects had a mean BMI of 24.2 $\pm 0.9 \mathrm{~kg} / \mathrm{m}^{2}$, a HOMA-R of $4.90 \pm 0.5$, and fasting insulin of $117 \pm 10 \mathrm{pmol} / 1$. Of $D Q B 1^{*} 0602$-positive subjects, $23 / 68$ $(33.8 \%)$ had fasting insulin values greater than $120 \mathrm{pmol} / 1$. Both the HOMA-R and the fasting insulin values in the subjects with $D Q B 1 * 0602$ were significantly greater than in the $D Q B 1^{*} 0602$-negative controls, while the mean BMI was not different between groups (Table 1).

As for measures reflective of beta cell function, the mean HOMA-B value was $219.2 \pm 15.2$ in $D Q B 1 * 0602$ positive subjects. This was not different from those without $D Q A 1 * 0102 / D Q B 1 * 0602$ (HOMA-B 191.5 \pm 9.8 , $p=0.1$ ). The mean FPIR of the study subjects with $D Q A 1 * 0102 / D Q B 1 * 0602$ was $1376 \pm 107$, with only $9 / 68$ $(13.2 \%)$ having FPIR below threshold. Among the 59 subjects with intact FPIR, 18 or $30.5 \%$ had abnormal glucose tolerance.

There was no relationship between the presence of autoantibodies, the occurrence of a high-risk second haplotype $(0301 / 0302$ or $0501 / 0201)$, or low FPIR and the existence of abnormal glucose tolerance, while those with abnormal glucose tolerance had higher HOMA-R values than those with normal glucose tolerance, though the latter was probably not significant due to multiple comparisons (Table 2).

Table 2 Relationship of autoantibodies, FPIR, and HOMA-R with glucose tolerance among $D Q B 1 * 0602$-positive subjects

\begin{tabular}{lcccccc}
\hline & GADA (\%) & ICA512A (\%) & mIAA (\%) & Low FPIR (\%) & HOMA-R (mean) & High-risk second allele \\
\hline Abnormal glucose tolerance $(n=22)$ & $22.7 \%$ & $0 \%$ & $13.6 \%$ & $18.2 \%$ & 6.79 & $59.1 \%$ \\
Normal glucose tolerance $(n=46)$ & $15.2 \%$ & $6.5 \%$ & $2.2 \%$ & $10.9 \%$ & 3.99 & $50.0 \%$ \\
$p$ value & $0.50^{\mathrm{a}}$ & $0.55^{\mathrm{a}}$ & $0.10^{\mathrm{a}}$ & $0.46^{\mathrm{a}}$ & $0.046^{\mathrm{b}}$ & $0.49^{\mathrm{a}}$ \\
\hline
\end{tabular}

$G A D$ glutamic acid decarboxylase, FPIR first-phase insulin release

${ }^{\text {a } B a s e d ~ o n ~ F i s h e r ' s ~ e x a c t ~ t e s t ~}$

${ }^{\mathrm{b}}$ Based on $t$-test 


\section{Discussion}

This study is the first to demonstrate that $D Q A 1 * 0102$ / $D Q B 1^{*} 0602$ relatives identified on an initial sample as ICA-positive often manifest abnormalities in glucose tolerance. Protection from type 1 diabetes has been previously reported in a small number of antibody-positive $D Q B 1^{*} 0602$ relatives [8-10]. Our data suggest that $D Q A 1 * 0102 / D Q B 1 * 0602$ does not provide protection from metabolic outcomes associated with the process of diabetes development (i.e. IGT/IFG), although this process may be somewhat atypical of type 1 diabetes. Indeed, we have shown that these subjects manifest a phenotype incorporating features of type 1 and type 2 diabetes. Specifically, the presence of autoantibodies commonly associated with type 1 diabetes and low first-phase insulin secretion are not associated with abnormalities of glucose tolerance among the $D Q B 1 * 0602$ relatives. A striking finding from our study is that these subjects had elevated fasting insulin levels. Elevated fasting insulin is often associated with the insulin resistance of type 2 diabetes. Indeed, HOMA-R, a measure of insulin resistance, was not only increased among the $D Q B 1^{*} 0602$-positive vs $D Q B 1^{*} 0602$-negative relatives, it was of a similar magnitude to that reported among type 2 patients [23], and greater than that seen among adolescents $[24,25]$. Interestingly, the marked difference in insulin resistance between the groups was not seen when BMI was tested, suggesting that obesity may not be the only factor contributing to the insulin resistance seen.

Our data show that this high frequency of abnormal glucose tolerance was greater than that seen in the $D Q A 1 * 0102 / D Q B 1 * 0602$-negative, but ICA-positive relatives. It is also greater than that seen in an older general population, where $22 \%$ of a cross-section of adults between 40 and 74 years had abnormal glucose tolerance or diabetes [26].

Since these observations suggested that these subjects were atypical of the type 1 disease process, we explored the possibility that the proband in the family also manifested an unusual form of diabetes. Instead we found the expected HLA types among family members with diabetes, suggesting that the typical type 1 diabetes disease process is present in their families. To explore this question further, we also obtained information about the presence of diabetes in other family members. Among all the families, $22 / 68$ reported diabetes in two generations and 2/68 in three generations. Though this proportion of multi-generation diabetes may suggest the possibility of MODY, it is important to keep in mind that DPT-1 selected for subjects with first- or second-degree relatives with diabetes and the number of multiplex families did not differ between the 0602-positive subjects and the 0602-negative matched control group (data not shown). In addition, neither the phenotype of abnormal glucose tolerance nor elevated fasting insulin identified families with distinct diabetes inheritance patterns. We also asked about the presence of type 2 diabetes in family members, since the insulin resistance suggested the possibility of co-existing type 2 diabetes genetic load. However, there was no increased frequency of type 2 diabetes among first- or second-degree family members by history (data not shown). Nevertheless, since type 2 diabetes is often not diagnosed, formal testing of this hypothesis requires metabolic testing of asymptomatic family members. This process, as well as HLA and autoantibody determination in these families is ongoing. Since it is difficult to propose a mechanism whereby HLA type in itself would affect insulin resistance, such an investigation can allow us to better understand the apparent relationship between $D Q B 1^{*} 0602$ and insulin resistance observed in this study.

Despite the high frequency of abnormal glucose tolerance in this group, it is still unknown whether these subjects will eventually progress to diabetes. Only two of those studied prospectively have diabetes according to OGTT criteria and associated with abnormal $\mathrm{HbA}_{1} \mathrm{c}$, and as previously reported, only one additional subject has reported development of diabetes. Among subjects enrolled in the DPT-1 intervention trials, the presence of IGT results in an approximately 70\%5-year risk of type 1 diabetes, as determined from life-table analysis [11]. A similar high risk is seen among people with IGT and at risk for type 2 diabetes, with about $6 \%$ annual progression [27, 28]. It is possible that many of the $D Q A 1 * 0102$ / $D Q B 1 * 0602$ subjects with abnormal glucose tolerance will either never manifest overt diabetes, or will develop diabetes only later in life. Such a scenario would be consistent with data indicating that the relative protection of $D Q A 1^{*} 0102 / D Q B 1^{*} 0602$ against the development of type 1 diabetes wanes with increasing age of diagnosis $[29,30]$. Similarly, the frequency of high-risk alleles is decreased with increasing age at diagnosis [31, 32]. Alternatively, a slow progression of disease may result in patients being clinically classified as having type 2 diabetes or latent autoimmune diabetes of adults (LADA) [33]. In this regard, it is important to note that $D Q B 1^{*} 0602$ may not protect subjects from LADA, as one report has shown that these patients have the same frequency of this allele as seen in the general population [27]. Others report no differences in the frequency of highrisk alleles between LADA and older, but more typical type 1 patients [34].

In this report, we have demonstrated for the first time that $D Q A 1 * 0102 / D Q B 1^{*} 0602$ relatives of type 1 patients identified at screening as being ICA-positive have a high frequency of abnormal glucose tolerance, whether or not ICA was confirmed positive on a subsequent sample. The lack of association between these glucose abnormalities and markers of autoimmunity emphasises the fact that we cannot determine from this study whether the screening of family members for ICA detected a selected group of $D Q A 1 * 0102 / D Q B 1 * 0602$ individuals, or whether the abnormal glucose tolerance is related to being an $D Q A 1 * 0102 / D Q B 1 * 0602$ relative of an individual with type 1 diabetes per se. Further studies are planned to address this question. Nonetheless, the presence of this haplotype in these subjects does not provide dominant protection from metabolic outcomes associated with the 
diabetes disease process, although this process may be better characterised as atypical of type 1 diabetes and more resembling type 2 diabetes or LADA. Our data indicate that multiple pathways to abnormal glucose tolerance are present within families of type 1 diabetic patients. The increasing recognition that some patients of all ages with diabetes are not clearly classifiable as either type 1 or type 2 suggests that a new paradigm incorporating the relationship between insulin secretion and resistance may further our understanding of the disease process and allow for a wider range of therapeutic options before and after diagnosis.

Acknowledgements This study was supported by NIAID R01 AI 44431-03 "DQB1*0602 Relatives-Mechanisms for Diabetes Protection". Subjects were identified by the Diabetes Prevention TrialType 1 Diabetes (DPT-1). The DPT-1 is supported through cooperative agreements by the Division of Diabetes, Endocrinology and Metabolic Diseases, National Institute of Diabetes and Digestive and Kidney Diseases, National Institutes of Health (NIH), and by the National Institute of Allergy and Infectious Disease, NIH; the National Institute of Child Health and Human Development, NIH; the National Center for Research Resources, NIH; the American Diabetes Association; the Juvenile Diabetes Research Foundation International; and various corporate sponsors. These investigations also relied upon the Clinical Research Centers at DPT-1 sites including the University of Washington (N01RR00037).

C.J. Greenbaum is supported in part by grants from the Paul G. Allen Foundation Clinical Scholars Program and the Buse Diabetes Clinical Research Chair. M. Atkinson's work was also supported by NIH awards 42288 and 39250.

\section{References}

1. Greenbaum CJ, Schatz DA, Cuthbertson D, Zeidler A, Eisenbarth GS, Krischer JP (2000) Islet cell antibody-positive relatives with human leukocyte antigen DQA1*0102, DQB $1 * 0602$ : identification by the diabetes prevention trialtype 1. J Clin Endocrinol Metab 85:1255-1260

2. Kockum I, Lernmark A, Dahlquist G et al (1996) Genetic and immunological findings in patients with newly diagnosed insulin-dependent diabetes mellitus. The Swedish Childhood Diabetes Study Group and the Diabetes Incidence in Sweden Study (DISS) Group. Horm Metab Res 28:344-347

3. Nepom GT (1993) Immunogenetics and IDDM. Diabetes Rev 1:93-103

4. Erlich HA, Griffith RL, Bugawan TL, Ziegler R, Alper C, Eisenbarth GS (1991) Implication of specific DQB1 alleles in genetic susceptibility and resistance by identification of IDDM siblings with novel HLA-DQB1 allele and unusual DR2 and DR1 haplotypes. Diabetes 40:478-481

5. Reijonen H, Ilonen J, Akerblom HK, Knip M, Dosch HM (1994) Multi-locus analysis of HLA class II genes in DR2positive IDDM haplotypes in Finland. The "Childhood Diabetes in Finland" (DiMe) Study Group. Tissue Antigens 43:1-6

6. Baisch JM, Weeks T, Giles R, Hoover M, Stastny P, Capra JD (1990) Analysis of HLA-DQ genotypes and susceptibility in insulin-dependent diabetes mellitus. N Engl J Med 322:18361841

7. Huang HS, Peng JT, She JY et al (1995) HLA-encoded susceptibility to insulin-dependent diabetes mellitus is determined by DR and DQ genes as well as their linkage disequilibria in a Chinese population. Hum Immunol 44:210 219
8. Pugliese A, Gianani R, Moromisato R (1995) HLA DQB1*0602 is associated with dominant protection from diabetes even among islet cell antibody positive first degree relatives of patients with insulin-dependent diabetes. Diabetes 44:608-613

9. Huang W, She JX, Muir A et al (1994) High risk HLA-DR/DQ genotypes for IDD confer susceptibility to autoantibodies but DQB1*0602 does not prevent them. J Autoimmun 7:889-897

10. Gianani R, Verge CF, Moromisato-Gianani RI et al (1996) Limited loss of tolerance to islet autoantigens in ICA+ first degree relatives of patients with type I diabetes expressing the HLA DQB1*0602 allele. J Autoimmun 9:723-725

11. Diabetes Prevention Trial Study Group (2002) Effects of insulin in relatives of patients with type 1 diabetes mellitus. N Engl J Med 346:1685-1691

12. Greenbaum CJ, Gaur LK, Noble JA (2002) ICA+ relatives with DQA $1 * 0102 / \mathrm{DQB} 1 * 0602$ have expected 0602 sequence and DR types. J Autoimmun 18:67-70

13. Verge CF, Stenger D, Bonifacio E et al (1998) Combined use of autoantibodies (IA-2 autoantibody, GAD autoantibody, insulin autoantibody, cytoplasmic islet cell antibodies) in type 1 diabetes: combinatorial islet autoantibody workshop. Diabetes 47:1857-1866

14. Yu L, Rewers M, Gianani R et al (1996) Anti-islet autoantibodies usually develop sequentially rather than simultaneously. J Clin Endocrinol Metab 81:4264-4267

15. Expert Committee on the Diagnosis and Classification of Diabetes Mellitus (1997) Report of the expert committee on the diagnosis and classification of diabetes mellitus. Diabetes Care 20:1183-1197

16. Bingley PJ, ICARUS Group (1996) Interactions of age, islet cell antibodies, insulin autoantibodies, and first-phase insulin response in predicting risk of progression to IDDM in ICA+ relatives: the ICARUS data set. Diabetes 45:1720-1728

17. McCulloch D, Bingley P, Colman P, Jackson R, Gale E (1993) Comparison of bolus and infusion protocols for determining acute insulin response to intravenous glucose in normal humans. Diabetes Care 16:911-915

18. Levy JC, Matthews DR, Hermans MP (1998) Correct homeostasis model assessment (HOMA) evaluation uses the computer program. Diabetes Care 21:2191-2192

19. Matthews DR, Hosker JP, Rudenski AS, Naylor BA, Treacher DF, Turner RC (1985) Homeostasis model assessment: insulin resistance and beta-cell function from fasting plasma glucose and insulin concentrations in man. Diabetologia 28:412-419

20. Haffner SM, Kennedy E, Gonzalez C, Stern MP, Miettinen H (1996) A prospective analysis of the HOMA model. The Mexico City Diabetes Study. Diabetes Care 19:1138-1141

21. Bugawan L, Erlich HA (1991) Rapid typing of HLA-DQB1 DNA polymorphism using nonradioactive oligonucleotide probes and amplified DNA. Immunogenetics 33:163-170

22. Gyllensten UB, Erlich HA (1988) Generation of singlestranded DNA by the polymerase chain reaction and its application to direct sequencing of the HLA-DQA locus. Proc Natl Acad Sci U S A 85:7652-7656

23. Bonora E, Targher G, Alberiche M et al (2000) Homeostasis model assessment closely mirrors the glucose clamp technique in the assessment of insulin sensitivity: studies in subjects with various degrees of glucose tolerance and insulin sensitivity. Diabetes Care 23:57-63

24. Frontini MG, Srinivasan SR, Berenson GS (2003) Longitudinal changes in risk variables underlying metabolic Syndrome X from childhood to young adulthood in female subjects with a history of early menarche: the Bogalusa Heart Study. Int J Obes Relat Metab Disord 27:1398-1404

25. Srinivasan SR, Frontini MG, Berenson GS (2003) Longitudinal changes in risk variables of insulin resistance syndrome from childhood to young adulthood in offspring of parents with type 2 diabetes: the Bogalusa Heart Study. Metabolism 52:443-450 
26. Harris MI, Flegal KM, Cowie CC et al (1998) Prevalence of diabetes, impaired fasting glucose, and impaired glucose tolerance in U.S. adults. The third national health and nutrition examination survey, 1988-1994. Diabetes Care 21:518-524

27. Tuomi T, Carlsson A, Li H et al (1999) Clinical and genetic characteristics of type 2 diabetes with and without GAD antibodies. Diabetes 48:150-157

28. Edelstein SL, Knowler WC, Bain RP et al (1997) Predictors of progression from impaired glucose tolerance to NIDDM: an analysis of six prospective studies. Diabetes 46:701-710

29. Caillat-Zucman S, Garchon HJ, Timsit J et al (1992) Agedependent HLA genetic heterogeneity of type 1 insulindependent diabetes mellitus. J Clin Invest 90:224-250

30. Graham J, Kockum I, Sanjeevi CB et al (1999) Negative association between type 1 diabetes and HLA DQB $1 * 0602$ DQA $1 * 0102$ is attenuated with age at onset. Swedish Childhood Diabetes Study Group. Eur J Immunogenet 26:117-127
31. Lohmann T, Sessler J, Verlohren HJ et al (1997) Distinct genetic and immunological features in patients with onset of IDDM before and after age 40. Diabetes Care 20:524-529

32. Horton V, Stratton I, Bottazzo GF et al (1999) Genetic heterogeneity of autoimmune diabetes: age of presentation in adults is influenced by HLA DRB1 and DQB1 genotypes (UKPDS 43). UK Prospective Diabetes Study (UKPDS) Group. Diabetologia 42:608-616

33. Groop LC, Bottazzo GF, Doniach D (1986) Islet cell antibodies identify latent type I diabetes in patients aged 35-75 years at diagnosis. Diabetes 35:237-241

34. Hosszufalusi N, Vatay A, Rajczy K et al (2003) Similar genetic features and different islet cell autoantibody pattern of latent autoimmune diabetes in adults (LADA) compared with adultonset type 1 diabetes with rapid progression. Diabetes Care $26: 452-457$ 